\title{
Preselection and Expert Advice*
}

\author{
Elisabeth SCHULTE ${ }^{\dagger}$ \\ Philipps-Universität Marburg
}

Mike FELGENHAUER

Plymouth University

August 11, 2016

\begin{abstract}
We study the effects of preselection on an expert's advice about the execution of a project if its execution yields more precise estimates about the expert's expertise. The introduction of a preselection stage, in which the decision maker evaluates the project before asking for advice, alters the expert's perception of the problem. We identify conditions under which preselection occurs in equilibrium. We show that if the expert adjusts his behavior, the option to preselect may reduce the expected utility of the decision maker.
\end{abstract}

Keywords: Reputation, cheap talk, safe haven.

JEL classification: D82; D83

\footnotetext{
${ }^{*}$ We thank Philippe Aghion, Jan Eeckhout, Georgy Egorov, Hans Peter Grüner, Oliver Kirchkamp, Benny Moldovanu, Nicola Persico, Ernst-Ludwig von Thadden, and the participants at seminars and conferences where this research has been presented for useful comments and suggestions. Elisabeth Schulte gratefully acknowledges support through the Collaborative Research Center 884.

${ }^{\dagger}$ Correspondence address: Elisabeth Schulte, Philipps-Universität Marburg, School of Business and Economics, Marburg Centre for Institutional Economics (MACIE), D-35032 Marburg, Germany; email: elisabeth.schulte@wiwi.uni-marburg.de; Tel: +496421 28230280.
} 


\section{Introduction}

In this paper we show that a decision maker's preselection of projects before consulting an expert can cause the quality of the expert's advice on the project that reaches him to deteriorate. As a consequence, the decision maker may be better off without acquiring information, but she is unable to commit to abstaining from information acquisition.

We consider a situation in which a decision maker has to choose whether to execute a project, facing uncertainty about the project's quality. She cares about making the appropriate decision and she may ask an expert with reputational concerns for advice. We assume that the ex post information about the quality of the expert's advice depends on whether the project is executed or not. E.g., the profit generated by a project can typically only be observed if it is implemented, but not if the project is not carried out. ${ }^{1}$ Reputational concerns may lead to biased advice in such a decision environment. Mediocre experts tend to favor the less informative alternative.

We show how the introduction of a preselection stage affects the expert's advice and we study the associated effects on the quality of the decision. Preselection means that the decision maker may privately evaluate the project and sometimes discard it without consulting the expert. That is, a preselection stage allows the decision maker to condition the consultation of the expert on her own information. Preselection is often used in practice. In firms, a set of projects is pre-screened on one management layer and only a subset is forwarded to the next one. In the academic publication process, many journals have desk rejection policies. Preselection allows the decision maker to economize on expert resources. It may also be the case that only with the observation of a first positive signal at the preselection stage consulting the expert becomes worthwhile.

Preselection alters the expert's decision problem. If he has to give advice on a preselected project, then he knows that the decision maker thinks that it is promising. He becomes more inclined to recommend the execution of the project.

Preselection can have adverse effects on the decision quality. Conditional on reaching the expert, a bad project may be executed with a higher probability due to the expert's strategic response to the information processing procedure. Preselection also increases the risk that a good project is rejected at the preselection stage. ${ }^{2}$

We identify a parameter region for which the decision maker applies a preselection policy in

\footnotetext{
${ }^{1}$ In a lobbying setting, Leaver (2009) shows that an interested party has an incentive to reveal the state of the world only for one type of decision in order to induce biased decision making.

${ }^{2}$ It is reasonable to assume that the decision maker has only limited time for pre-screening. Note also that if the decision maker has perfect information about the project's quality, then the expert's advice is superfluous.
} 
order to reduce the expected cost of asking for advice, but the expert's strategic adjustment to this policy is excessively detrimental. The decision maker would be better off if she could commit not to preselect at all. However, as the expert cannot observe the decision maker's information acquisition choice, he anticipates that the decision maker will preselect in equilibrium and behaves accordingly.

\section{Related literature}

Our paper contributes to the literature on information processing when the experts have reputational concerns. The seminal papers on reputational concerns and their effects on expert advice are Holmström (1999) and Scharfstein and Stein (1990). In both papers, there is uncertainty but no asymmetric information about the expert's expertise. In Scharfstein and Stein (1990), the experts' information is correlated and reputational concerns lead to herding behavior. ${ }^{3}$ In Holmström's paper, output is an indicator for expertise and reputational concerns deter investment in profitable opportunities and distort working incentives. With private information on the agent's side as in Chen (2015), the investment choice can serve as a signaling device and reputational concerns cause overinvestment in a risky asset. Likewise, in the herding setup private information about expertise can prevent herding and may cause anti-herding (e.g., Avery and Chevalier, 1999, Effinger and Polborn, 2001, and Levy, 2004). It is common in this literature to assume that the expert is directly interested in a reputation for expertise. ${ }^{4}$ To this literature, we add the consideration of two information sources for the decision maker, to acquire information on her own and/or to consult an expert with reputational concerns for advice. We show that the access to the former source may deteriorate the value of the second source sufficiently such that the decision maker is better off without access to it.

Swank and Visser (2008) study a setting of sequential decision making in which both decision makers' expertise is endogenous, and both have reputational concerns. With costly information acquisition, the herding problem can become a free-rider problem. In our setting, the first decision maker has no reputational concerns, information acquisition is costly for her at both stages and the consultation of the expert is endogenous. In our model, the decision maker's

\footnotetext{
${ }^{3}$ See also Ottaviani and Sørensen (2001). In Ottaviani and Sørensen (2006), they show that reputational concerns yield a bias towards reporting the prior mean in a continuous version of the model in which information about the state is revealed ex post. They show that in equilibrium, information transmission is binary.

${ }^{4}$ In Sobel (1985), Bénabou and Laroque (1992), Morris (2001) and Lagerlöf and Frisell (2007), there is an instrumental preference for a reputation for honesty, which increases the sender's impact on the decisions in later periods.
} 
motive to acquire a costly signal is either to sometimes save the cost associated with consulting the expert or to make consulting the expert worthwhile.

Fox and Van Weelden (2010) also consider a reputational cheap talk model of two sequentially moving, imperfectly informed experts. In their model, disagreement hurts both experts' reputations. The effects of a preference for the other expert's reputation (partisanship) on the second expert's information revelation incentives and on the decision quality are studied. In our analysis, the first mover's expertise is endogenous private information, and we study the feedback of information acquisition in the first stage on the second expert's information revelation incentives and the decision quality.

Suurmond, Visser and Swank (2004) show that reputational concerns lead to biased advice, but may increase information acquisition incentives. They find that both effects are stronger in a fully transparent decision situation than if ex post information is decision-dependent. In Milbourn et al. (2001), reputational concerns boost the expert's information acquisition incentives even without a distortion in reports. ${ }^{5}$

In Levy (2005) ex post revelation of information is endogenous. Some decisions are more likely to be double-checked than others. In Levy's setting, asymmetric ex post revelation of information mitigates the incentive to contradict the (exogenous) prior in order to signal ability. In our paper instead, there is an exogenous asymmetry in the informativeness of the decision as in Suurmond, Visser and Swank (2004), but the expert's prior depends on the decision maker's preselection behavior. The preselection mechanism provides a counterbalance to the bias induced by the asymmetry in ex post information revelation and increases the attractiveness of recommending the execution of the project. Similarly, in Liu and Sanyal (2012) the option for the decision maker to revert to the status quo upon the arrival of interim news causes a mediocre expert to recommend the implementation of the alternative with better ex post information more aggressively. The decision maker may be better off without the availability of interim news.

$\mathrm{Fu}$ and $\mathrm{Li}$ (2014) analyze the effect of the institutional environment on a career-concerned agent's incentive to implement a reform. In contrast to our model, a low ability agent takes an

\footnotetext{
${ }^{5}$ A similar effect arises in Kartik, Lee and Suen (forthcoming). They study a persuasion game in which experts' expertise depends on their information acquisition efforts. In their paper, experts are only interested in the decision maker's belief regarding the state of the world. Because there is an option to hide unfavorable information (but no possibility to verify the absence of information), a "reputation" for expertise is harmful for an expert who does not possess favorable information. If the decision maker believes that an expert has a high expertise, the expert has a strong incentive to acquire information.
} 
inefficient risky action too often. A conservative institution reduces this incentive but comes at the cost that the high ability agent is prevented from conducting efficient reforms.

Prat (2005) shows that transparency may be bad for the decision maker. In his model, if the decision maker cannot observe the expert's actions, they reflect the correlation between the expert's information and the state. If the decision maker can observe the expert's actions, they reflect the correlation between the signal and the expert's type (about which players are symmetrically informed). In our model, the decision maker can choose to become (imperfectly) informed prior to consulting the expert. As the expert anticipates that the decision maker is informed, he uses the correlation between the decision maker's signal and the state, which can dilute the informativeness of his advice.

\section{Model}

The decision maker has the choice between executing and rejecting a project. Denote her choice by $x_{d} \in\{Y, N\}$. The project's quality is either good or bad, $\omega \in\{g, b\}$. Let $w$ denote the prior probability that the project is good. The decision maker strictly prefers to carry out the project $\left(x_{d}=Y\right)$ if it is good, and to reject the project $\left(x_{d}=N\right)$ if it is bad. Denote her utility with $u\left(x_{d}, \omega\right)$. The decision maker seeks to maximize her expected utility net of the expected cost of information.

She can acquire information from two different sources: She can observe an imperfect private signal $\sigma_{d} \in\{g, b\}$, with $\operatorname{prob}\left\{\sigma_{d}=g \mid \omega=g\right\}=\operatorname{prob}\left\{\sigma_{d}=b \mid \omega=b\right\}=p_{d} \in(1 / 2,1)$. Acquiring the signal $\sigma_{d}$ comes at a cost $c_{d}$ for the decision maker. Denote the event that the decision maker does not observe the signal by $\sigma_{d}=\emptyset$.

In addition, and independently of the decision whether to acquire $\sigma_{d}$, the decision maker can consult an expert. Consulting the expert comes at a cost $c_{e}$ for the decision maker. The expert is either smart or mediocre, $\theta \in\{s, m\}$. The expert privately observes his type $\theta$ and a signal $\sigma_{\theta} \in\{g, b\}$ with $\operatorname{prob}\left\{\sigma_{\theta}=g \mid \omega=g\right\}=\operatorname{prob}\left\{\sigma_{\theta}=b \mid \omega=b\right\}=p_{\theta}$. We denote the prior probability that the expert is smart with $q$ and assume that $p_{s}=1$ and $p_{m} \in(1 / 2,1) .{ }^{6}$

The expert's task is to formulate a recommendation (a cheap talk message) whether the

\footnotetext{
${ }^{6}$ In the online appendix to this paper we study the expert's equilibrium behavior for $p_{s} \in(1 / 2,1], p_{s}>$ $p_{m}$. The assumption that the smart expert correctly observes the true quality simplifies notation, avoids case distincitions, and gives an unambiguous meaning to the notion of "sincere advice". If the expert did not know the true state of the world with certainty, a sincere recommendation would depend on the decision maker's preferences and on her own information.
} 
project shall be carried out or not, $x_{e} \in\{Y, N\} .{ }^{7}$ His strategy maps his type and his private signal into a probability to recommend the execution of the project. We denote with $\gamma_{\theta}$ the probability that the expert of type $\theta$ with signal $\sigma_{\theta}=g$ recommends to carry out the project, $\gamma_{\theta}=\operatorname{prob}\left\{x_{e}=Y \mid \theta, \sigma_{\theta}=g\right\} . \beta_{\theta}$ denotes the probability that the expert of type $\theta$ with signal $\sigma_{\theta}=b$ recommends to reject the project, $\beta_{\theta}=\operatorname{prob}\left\{x_{e}=N \mid \theta, \sigma_{\theta}=b\right\}$. The expert's objective is to appear smart to the decision maker. He seeks to maximize his expected reputation $\mathbb{E}[\hat{q}]$, where $\hat{q}$ is the decision maker's posterior assessment of the probability that the expert is smart, taking into account all the information available to her.

Our equilibrium concept is sequential equilibrium (Kreps and Wilson, 1982). We focus attention on equilibria in which the smart expert provides sincere advice, i.e., $\gamma_{s}=\beta_{s}=1$. A full analysis of the expert's possible equilibrium behavior is presented in the online appendix to this paper. We argue that giving sincere advice is the most plausible behavior on part of the smart expert. If the decision maker expected babbling, she would not consult the expert in equilibrium. In the following, the notion of equilibrium refers to the selected one. Consequently, as the smart type's behavior is fixed, the discussion of the expert's behavior refers to the behavior of the mediocre type. The timing of events is as follows:

1. Nature chooses $\omega$ and $\theta$. Conditional on that, nature chooses $\sigma_{d}$ and $\sigma_{\theta}$.

2. The decision maker decides whether to acquire signal $\sigma_{d}$ at $\operatorname{cost} c_{d}$.

3. The decision maker decides whether to consult the expert at cost $c_{e}$.

4. The expert observes $\theta$ and $\sigma_{\theta}$ and provides a recommendation if consulted.

5. The decision maker decides whether to carry out the project.

6. If the project is executed, $\omega$ is revealed.

We assume that ex ante, without access to further information, the decision maker prefers to reject the project, and that she still prefers to reject the project even conditional on observing $\sigma_{d}=g$ (but before consulting the expert). Under this assumption, which jointly restricts the parameter spaces for the prior, the decision maker's preferences and the precision of her signal, the only purpose of acquiring the signal $\sigma_{d}$ is to condition the consultation of the expert on its realization. For the decision maker to be responsive to information at all, either the probability that she faces the smart expert must be sufficiently high, or the mediocre expert's signal must be sufficiently precise and his report must be sufficiently informative. We assume throughout

\footnotetext{
${ }^{7}$ We model the expert's recommendation as cheap talk, as experts usually make a fair amount of subjective assessments and summary recommendations. Though it is common to back up a recommendation with arguments, we think that it is usually possible to find arguments for either recommendation (see Felgenhauer and Schulte, 2014).
} 
that $q>1 / 2$. We further restrict the parameter range under consideration in Section 3.3, where the context of the restriction becomes clear.

We focus attention on pure signal acquisition behavior on part of the decision maker, ${ }^{8}$ and we call an equilibrium in which the decision maker decides to acquire the signal $\sigma_{d}$ an equilibrium with preselection. An equilibrium in which the decision maker does not acquire the signal is called an equilibrium without preselection. We assume that the expert cannot observe the decision maker's signal acquisition.

\section{Analysis}

We work backwards through the stages of the game. Due to the ex-post asymmetry in information revelation, we first need to understand under which circumstances the project is implemented before we can study the expert's incentives for giving advice. Hence, we start our analysis with the derivation of the decision maker's behavior regarding the project implementation. The expert in equilibrium incorporates any information he can deduce from the decision maker's consultation behavior, and the decision maker's benefits of consulting the expert depends on his reporting behavior. Thus, we next jointly analyse the decision maker's optimal consultation of the expert and the expert's optimal advice, taking as given the decision maker's previous signal acquisition decision and anticipating her behavior at the stage of project choice. We then study the equilibrium pattern of preselection. Having specified the players' optimal behavior at all stages of the game, we draw conclusions about the decision quality.

\subsection{Project choice}

At the stage of making the project choice, all information acquisition costs are sunk and can be neglected. Denote the decision maker's posterior that the project is of good quality with $\hat{w}$. If she decides to carry out the project, her expected utility is $\hat{w} u(Y, g)+(1-\hat{w}) u(Y, b)$. If she decides to reject the project, her expected utility is $\hat{w} u(N, g)+(1-\hat{w}) u(N, b)$.

Denote with $\Delta_{g}$ the decision maker's gain in utility if instead of wrongfully rejecting a project of good quality, the decision maker makes the utility-maximizing choice to carry the project out, i.e., $\Delta_{g}=u(Y, g)-u(N, g)$. Likewise, let $\Delta_{b}$ denote the decision maker's gain in utility if instead of wrongfully executing a bad project, the decision maker rejects it, i.e., $\Delta_{b}=u(N, b)-u(Y, b)$. It is optimal for the decision maker to carry out the project if and only

\footnotetext{
${ }^{8}$ We comment on this assumption in the appendix.
} 


$$
\hat{w} \geq \frac{\Delta_{b}}{\Delta_{b}+\Delta_{g}} .
$$

Let $\hat{w}^{\sigma_{d}}=\operatorname{prob}\left\{\omega=g \mid \sigma_{d}\right\}$ for $\sigma_{d} \in\{g, b, \emptyset\}$. As noted in the model description, we study the model for parameter constellations such that $\hat{w}^{g}<\frac{\Delta_{b}}{\Delta_{b}+\Delta_{g}}$, i.e., even if her own signal is good, the decision maker does not approve the project without a consultation of the expert.

\subsection{Expert consultation and advice}

As the expert's advice is costly to obtain, the decision maker consults the expert only if the project choice depends on the advice. Denote with $\hat{w}_{Y}^{\sigma_{d}}, \hat{w}_{N}^{\sigma_{d}}$ the decision maker's posterior given her own signal $\sigma_{d}$ and the expert's recommendation to execute $\left(x_{e}=Y\right)$ and to reject the project $\left(x_{e}=N\right)$, respectively. Recall that the smart expert provides sincere advice and that ex ante the decision maker assigns a higher probability to the smart type than to the mediocre type $(q>1 / 2)$. Consequently, regardless of the mediocre type's behavior, the decision maker is more optimistic about the project's quality when the expert recommends to carry out the project than when he recommends to reject it, i.e., $\hat{w}_{Y}^{\sigma_{d}}>\hat{w}_{N}^{\sigma_{d}}$. Two conclusions follow:

Lemma 1 (i) On the equilibrium path, the expert is consulted only if the consultation gives rise to a lottery over the decision maker's possible posteriors such that $\hat{w}_{Y}^{\sigma_{d}}>\Delta_{b} /\left(\Delta_{b}+\Delta_{g}\right)>\hat{w}_{N}^{\sigma_{d}}$. (ii) The decision maker follows the expert's advice if she asks for it.

The decision maker's own signal alone cannot convince her to execute the project. If she acquires an own signal, she does so in order to make the decision whether to ask for the expert's advice contingent on the signal realization.

Lemma 2 In an equilibrium with preselection, the decision maker consults the expert if and only if $\sigma_{d}=g$.

When deciding which recommendation to give, the mediocre expert faces a lottery. If he recommends to execute the project, the project is indeed executed. The project quality becomes apparent. If it turns out to be bad, the expert's mediocrity is revealed. However, if the project quality turns out to be good, this boosts the expert's reputation. The probability to obtain a high reputation depends on the expert's signal and on his belief about the decision maker's signal conditional on being consulted for advice. On the other hand, if the expert recommends to reject the project, the decision maker follows his advice and nothing more is learnt about 
the project's quality. In this sense, recommending to reject the project is the safe haven option for the mediocre expert.

Ceteris paribus, the more often the mediocre expert recommends to execute the project, the lower the reputation associated with this recommendation, and the higher the reputation associated with the recommendation to reject the project. This monotonicity guarantees the uniqueness of the mediocre expert's best response. Moreover, the higher the expert's reputation is ex ante, the more attractive it is for the mediocre type to pool in the safe haven.

Proposition 1 (i) The expert's equilibrium behavior is unique in a putative equilibrium with preselection and it is unique in a putative equilibrium without preselection.

(ii) There are $\underline{q}, \bar{q}$ and $\tilde{q}$ such that the mediocre expert gives sincere advice if and only if $q \in(\underline{q}, \bar{q}]$. If $q \leq \underline{q}, \gamma_{m}=1, \beta_{m}<1$. If $q>\bar{q}, \beta_{m}=1, \gamma_{m}<1$. If $q>\tilde{q}, \beta_{m}=1, \gamma_{m}=0$.

The thresholds stated in the above proposition depend on the expert's belief about whether the decision maker preselects. In a (putative) equilibrium with preselection, the expert's incentive to recommend the execution of the project is stronger than in a (putative) equilibrium without preselection, because the expert deduces the decision maker's good signal. He becomes more optimistic about the project's quality and, hence, he becomes more optimistic that the recommendation to execute the project yields a high reputation as the advice turns out to be correct. At the same time, the recommendation to reject the project yields a lower reputation, because it contradicts the decision maker's own signal. Consequently, in an equilibrium with preselection $\gamma_{m}$ is weakly higher and $\beta_{m}$ is weakly lower.

\subsection{Restricting the parameter range}

Previously, we have identified the general pattern of the interaction between the decision maker and the expert. Our main objective is to show that the option to preselect can be harmful for the decision maker. In order to avoid further case distinctions, we focus our analysis on a parameter range such that the expert truthfully reveals his signal in an equilibrium without preselection.

If there is no preselection, conditional on being asked for advice, the expert believes that the decision maker assigns the prior probability $w$ to $\omega=g$. Giving sincere advice, i.e., $\gamma_{m}=\beta_{m}=1$, is a best response if $q \in\left(\frac{w^{2}-(1-w)^{2}\left(\frac{p_{m}}{1-p_{m}}\right)^{2}}{w^{2}+(1-w)^{2}\left(\frac{p_{m}}{1-p_{m}}\right)}, \frac{w^{2}-(1-w)^{2}}{w^{2}+(1-w)^{2}\left(\frac{1-p_{m}}{p_{m}}\right)}\right]$, as we show in the proof of Proposition 1 (ii). In the appendix, we identify a set of parameter constellations for which the interval is non-empty. 
We further restrict the parameter space and concentrate on the case in which the expert's advice, given a sincere recommendation by both types, is sufficiently informative such that it is optimal for the decision maker to follow the advice if she does not acquire an own signal. Thus, parameters are such that the probability that the project is good conditional on receiving the advice to execute it exceeds $\frac{\Delta_{b}}{\Delta_{b}+\Delta_{g}}($ see $(1))$, i.e.,

$$
\begin{array}{r}
\frac{w(q+}{w\left(q+(1-q) p_{m}\right)} \geq \frac{\Delta_{b}}{\Delta_{b}+\Delta_{g}}+(1-w)(1-q)\left(1-p_{m}\right) \\
\Leftrightarrow \Delta_{b} \leq \frac{w\left(q+(1-q) p_{m}\right)}{(1-w)(1-q)\left(1-p_{m}\right)} \Delta_{g} .
\end{array}
$$

\subsection{The expert's behavior in an equilibrium with preselection}

The parameter range is chosen such that $\gamma_{m}=\beta_{m}=1$ if the decision maker does not preselect. In an equilibrium with preselection, the expert infers that the decision maker has observed a good signal if he is asked for advice. He is more optimistic about the project's quality than in an equilibrium without preselection. Hence, for the parameter range under consideration, $\gamma_{m}=1$. $\beta_{m}=1$ if and only if $q>\frac{\left(\hat{w}^{g}\right)^{2}-\left(1-\hat{w}^{g}\right)^{2}\left(\frac{p_{m}}{1-p_{m}}\right)^{2}}{\left(\hat{w}^{g}\right)^{2}+\left(1-\hat{w}^{g}\right)^{2}\left(\frac{p_{m}}{1-p_{m}}\right)}$. Otherwise, $\beta_{m}<1$. If the decision maker believes that the mediocre expert recommends the execution of the project with probability 1 if he has observed a good signal and with probability $\left(1-\hat{\beta}_{m}\right)$ if he has observed a bad signal, the reputation associated with having recommended a project that turns out to be good is $\frac{q}{q+(1-q)\left(p_{m}+\left(1-p_{m}\right)\left(1-\hat{\beta}_{m}\right)\right)}$. Conditional on the expert's bad signal and the decision maker's inferred good signal, the probability that the project is good is $\frac{\hat{w}^{g}\left(1-p_{m}\right)}{\hat{w}^{g}\left(1-p_{m}\right)+\left(1-\hat{w}^{g}\right) p_{m}}$. If the expert recommends to reject the project, his reputation is $\frac{q\left(1-\hat{w}^{g}\right)}{q\left(1-\hat{w}^{g}\right)+(1-q)\left(\left(1-\hat{w}^{g}\right) p_{m}+\hat{w}^{g}\left(1-p_{m}\right)\right) \hat{\beta}_{m}}$. The expert is indifferent between both recommendations if

$$
\frac{\hat{w}^{g}\left(1-p_{m}\right)}{\hat{w}^{g}\left(1-p_{m}\right)+\left(1-\hat{w}^{g}\right) p_{m}} \frac{q}{q+(1-q)\left(p_{m}+\left(1-p_{m}\right)\left(1-\hat{\beta}_{m}\right)\right)}=\frac{q\left(1-\hat{w}^{g}\right)}{q\left(1-\hat{w}^{g}\right)+(1-q)\left(\left(1-\hat{w}^{g}\right) p_{m}+\hat{w}^{g}\left(1-p_{m}\right)\right) \hat{\beta}_{m}} .
$$

The left-hand-side of the above equation increases in $\hat{\beta}_{m}$, the right-hand-side decreases in $\hat{\beta}_{m}$. Hence, there is at most one value for $\hat{\beta}_{m}$ for which the equation holds.

If $q>\frac{\left(\hat{w}^{g}\right)^{2}-\left(1-\hat{w}^{g}\right)^{2}\left(\frac{p_{m}}{1-p_{m}}\right)^{2}}{\left(\hat{w}^{g}\right)^{2}+\left(1-\hat{w}^{g}\right)^{2}\left(\frac{p_{m}}{1-p_{m}}\right)}, \gamma_{m}=\beta_{m}=1$ in an equilibrium with preselection and in an equilibrium without preselection. If $q \leq \frac{\left(\hat{w}^{g}\right)^{2}-\left(1-\hat{w}^{g}\right)^{2}\left(\frac{p_{m}}{1-p_{m}}\right)^{2}}{\left(\hat{w}^{g}\right)^{2}+\left(1-\hat{w}^{g}\right)^{2}\left(\frac{p_{m}}{1-p_{m}}\right)}$, in an equilibrium with preselection, the expert's optimal behavior $\beta_{m}^{*}$ solves $(2), \beta_{m}^{*}<1$.

\subsection{The value of information}

The next step is to derive the value of accessing the two sources of information, the decision maker's own signal and the expert's advice. Both are valuable only if the decision whether 
to execute the project depends on the realizations in a non-trivial manner. In order to avoid qualifications in our statements, we assume that the decision maker does not acquire information if she is indifferent (neither her own signal nor the expert's advice). ${ }^{9}$

\subsubsection{The value of the expert's advice}

The value of the expert's advice depends on the decision that the decision maker would take without asking for advice. Without access to the expert's advice, the decision maker prefers to reject the project. Her own signal alone cannot boost her assessment of the project's quality over the threshold for acceptance. If she assigns probability $\hat{w}^{\sigma_{d}}$ to $\omega=g$ and probability $\hat{\beta}_{m}$ to the mediocre expert truthfully revealing a bad signal, ${ }^{10}$ the (perceived) value of the expert's advice is:

$$
\begin{aligned}
V\left(\hat{w}^{\sigma_{d}}, \hat{\beta}_{m}\right):= & \hat{w}^{\sigma_{d}}\left(q+(1-q) p_{m}\right) \Delta_{g}-\left(1-\hat{w}^{\sigma_{d}}\right)(1-q)\left(1-p_{m}\right) \Delta_{b} \\
& +\left(1-\hat{\beta}_{m}\right)(1-q)\left(\hat{w}^{\sigma_{d}}\left(1-p_{m}\right) \Delta_{g}-\left(1-\hat{w}^{\sigma_{d}}\right) p_{m} \Delta_{b}\right) .
\end{aligned}
$$

An explicit derivation of (3) can be found in the appendix. $V\left(\hat{w}^{\sigma_{d}}, \hat{\beta}_{m}\right)>0$ implies that it is optimal for the decision maker to follow the expert's advice (conditional on having acquired it). If $V\left(\hat{w}^{\sigma_{d}}, \hat{\beta}_{m}\right)>c_{e}$, it is optimal for her to acquire his advice. In the parameter range under consideration, $V\left(\hat{w}^{\sigma_{d}}, \hat{\beta}_{m}\right)$ increases in $\hat{w}^{\sigma_{d}}$ and increases in $\hat{\beta}_{m}$ : The expert's advice is the more valuable, the more likely the decision maker considers a change in her decision, and the more likely the advice on which that change is based is correct. ${ }^{11}$

Lemma 3 Consider the restricted parameter range. The value of the expert's signal increases in $\hat{\beta}_{m}$.

\subsubsection{The value of the decision maker's signal}

The value of the decision maker's signal is positive if and only if it is optimal for her to ask for the expert's advice if $\sigma_{d}=g$, but not if $\sigma_{d}=b$ (see Lemma 2). It is optimal for her to ask for the expert's advice if and only if $c_{e}<V\left(\hat{w}^{\sigma_{d}}, \hat{\beta}_{m}\right)$. We have $V\left(\hat{w}^{b}, \hat{\beta}_{m}\right)<V\left(w, \hat{\beta}_{m}\right)<V\left(\hat{w}^{g}, \hat{\beta}_{m}\right)$. Consider Figure 1. If $c_{e}<V\left(\hat{w}^{b}, \hat{\beta}_{m}\right)$, in parameter range I, the decision maker anticipates that she will ask for advice regardless of the realization of her signal, such that the value of her own

\footnotetext{
${ }^{9}$ See our remark on mixed strategies in the appendix.

${ }^{10}$ Recall that for the parameter range under consideration, $\gamma_{m}=1$.

${ }^{11}$ At the same time, the higher $\beta_{m}$, the less likely the expert's advice to reject the project is correct. This is so because this recommendation then more often comes from the mediocre expert, and the mediocre expert's signal is less informative. Thus, this point requires an assumption on the decision maker's preferences.
} 
region I

expert advice is so cheap

that the decision maker

always asks for it

$\Rightarrow$ her own signal is worthless

$$
V\left(\hat{w}^{b}, \hat{\beta}_{m}\right) V\left(w, \hat{\beta}_{m}\right) \quad V\left(\hat{w}^{g}, \hat{\beta}_{m}\right)
$$

expert advice is so expensive

that the decision maker

never asks for it

$\Rightarrow$ her own signal is worthless

Figure 1: Relating the value of the decision maker's signal to the value of the expert's advice: Preselection can only occur in parameter region II

signal is zero. If $c_{e} \geq V\left(\hat{w}^{g}, \hat{\beta}_{m}\right)$ (parameter region III), she anticipates that she will never ask for the expert's advice, and again the value of her own signal is zero. For the remaining values of $c_{e}$ (parameter region II), we need to distinguish two cases, $c_{e}<V\left(w, \hat{\beta}_{m}\right)$ and $c_{e} \geq V\left(w, \hat{\beta}_{m}\right)$.

If the decision maker does not acquire an own signal, and $c_{e}<V\left(w, \hat{\beta}_{m}\right)$, it is optimal to ask for the expert's advice. The purpose of her own signal acquisition is to sometimes avoid the cost of asking for advice (if $\sigma_{d}=b$ ). If $\omega=g$, she forgoes the chance to revise her wrong decision if she does not ask for advice. If $\omega=b$, she sticks with the correct decision if she does not ask for advice. Considering all these effects, the value of her signal is

$$
\begin{array}{r}
\max \left\{0, w\left(1-p_{d}\right)\left(c_{e}-\left(q+(1-q)\left(p_{m}+\left(1-p_{m}\right)\left(1-\hat{\beta}_{m}\right)\right)\right) \Delta_{g}\right)\right. \\
\left.+(1-w) p_{d}\left(c_{e}+(1-q)\left(1-p_{m}+p_{m}\left(1-\hat{\beta}_{m}\right)\right) \Delta_{b}\right)\right\}
\end{array}
$$

(4) is zero for $c_{e} \leq V\left(\hat{w}^{b}, \hat{\beta}_{m}\right)$ and it is increasing in $c_{e}$ for $c_{e}>V\left(\hat{w}^{b}, \hat{\beta}_{m}\right)$.

If the decision maker does not acquire an own signal, and $c_{e} \geq V\left(w, \hat{\beta}_{m}\right)$, it is optimal not to ask for the expert's advice and to reject the project. The purpose of the decision maker's own signal acquisition is to make advice-seeking worthwhile (if $\sigma_{d}=g$ ). If $\omega=g$, she benefits from the chance to revise her wrong decision if she asks for advice. If $\omega=b$, she may revise her correct decision if she asks for the expert's advice. Considering all these effects, the value of her signal is

$$
\begin{aligned}
& \max \left\{0, w p_{d}\left(q+(1-q)\left(p_{m}+\left(1-p_{m}\right)\left(1-\hat{\beta}_{m}\right)\right) \Delta_{g}-c_{e}\right)\right. \\
& \left.-(1-w)\left(1-p_{d}\right)\left((1-q)\left(1-p_{m}+p_{m}\left(1-\hat{\beta}_{m}\right)\right) \Delta_{b}+c_{e}\right)\right\}
\end{aligned}
$$

(5) is zero for $c_{e} \geq V\left(\hat{w}^{g}, \hat{\beta}_{m}\right)$ and it is decreasing in $c_{e}$ for $c_{e}<V\left(\hat{w}^{g}, \hat{\beta}_{m}\right)$.

Denote with $\Phi\left(\hat{\beta}_{m}, c_{e}\right)$ the value of the decision maker's signal. It is the minimum of (4) and (5) for $c_{e} \in\left(V\left(\hat{w}^{b}, \hat{\beta}_{m}\right), V\left(\hat{w}^{g}, \hat{\beta}_{m}\right)\right)$, and zero outside this range. (4) $=(5)$ if $c_{e}=V\left(w, \hat{\beta}_{m}\right)$, see Figure 2. 


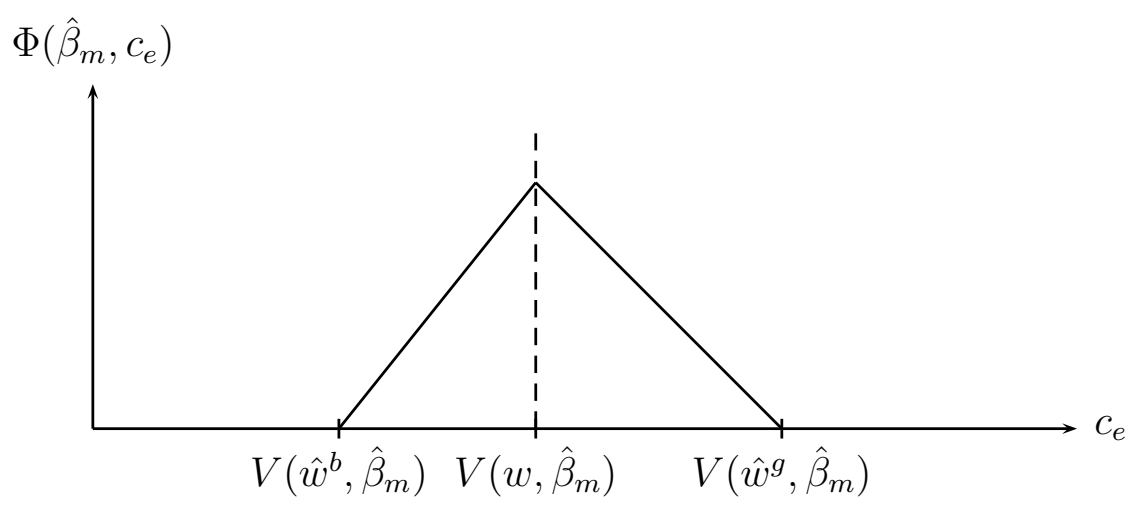

Figure 2: The solid triangle depicts the value of the decision maker's signal in the range where it is positive. For parameter constellations below the graph, preselection occurs in equilibrium, provided that the expert's advice does not depend on the preselection behavior. To the left of the dashed line, the decision maker always asks for advice if she does not preselect, to the right of the dashed line, she rejects the project straight away if she does not ask for advice.

The value of the expert's advice decreases if $\hat{\beta}_{m}$ decreases. If the decision maker's own signal acquisition is aimed at avoiding the cost of advice sometimes, its value is the higher, the lower the value of advice. If the decision maker's own signal acquisition is aimed at inducing advice-seeking sometimes, its value is the higher, the higher the value of advice.

Proposition 2 Consider the restricted parameter range and $c_{e} \in\left[V\left(\hat{w}^{b}, \hat{\beta}_{m}\right), V\left(\hat{w}^{g}, \hat{\beta}_{m}\right)\right)$.

(i) If $c_{e}<V\left(w, \hat{\beta}_{m}\right)$, then $\Phi\left(\hat{\beta}_{m}, c_{e}\right)$ decreases in $\hat{\beta}_{m}$.

(ii) If $c_{e}>V\left(w, \hat{\beta}_{m}\right)$, then $\Phi\left(\hat{\beta}_{m}, c_{e}\right)$ increases in $\hat{\beta}_{m}$.

\subsection{Equilibria}

We distinguish two parameter regions. If $q>\frac{\left(\hat{w}^{g}\right)^{2}-\left(1-\hat{w}^{g}\right)^{2}\left(\frac{p_{m}}{1-p_{m}}\right)^{2}}{\left(\hat{w}^{g}\right)^{2}+\left(1-\hat{w}^{g}\right)^{2}\left(\frac{p_{m}}{1-p_{m}}\right)}$, it is optimal for the expert to give sincere advice, independently of the decision maker's preselection behavior. Hence, the previous steps of our analysis characterize the equilibrium. There is a unique equilibrium for all parameter constellations, as illustrated in Figure 2 and described in the following proposition.

Proposition 3 Consider $q>\frac{\left(\hat{w}^{g}\right)^{2}-\left(1-\hat{w}^{g}\right)^{2}\left(\frac{p_{m}}{1-p_{m}}\right)^{2}}{\left(\hat{w}^{g}\right)^{2}+\left(1-\hat{w}^{g}\right)^{2}\left(\frac{p_{m}}{1-p_{m}}\right)}$. The expert gives sincere advice.

(i) If $c_{e}<V\left(\hat{w}^{b}, 1\right)$, the equilibrium is without preselection. The decision maker asks for the expert's advice.

(ii) If $c_{e} \in\left[V\left(\hat{w}^{b}, 1\right), V(w, 1)\right]$, the equilibrium is with preselection if $c_{d}<\Phi\left(1, c_{e}\right)$. Otherwise, the decision maker does not preselect and always asks for advice.

(iii) If $c_{e} \in\left[V(w, 1), V\left(\hat{w}^{g}, 1\right)\right)$, the equilibrium is with preselection if $c_{d}<\Phi\left(1, c_{e}\right)$. Otherwise, the decision maker rejects the project without acquiring any information. 


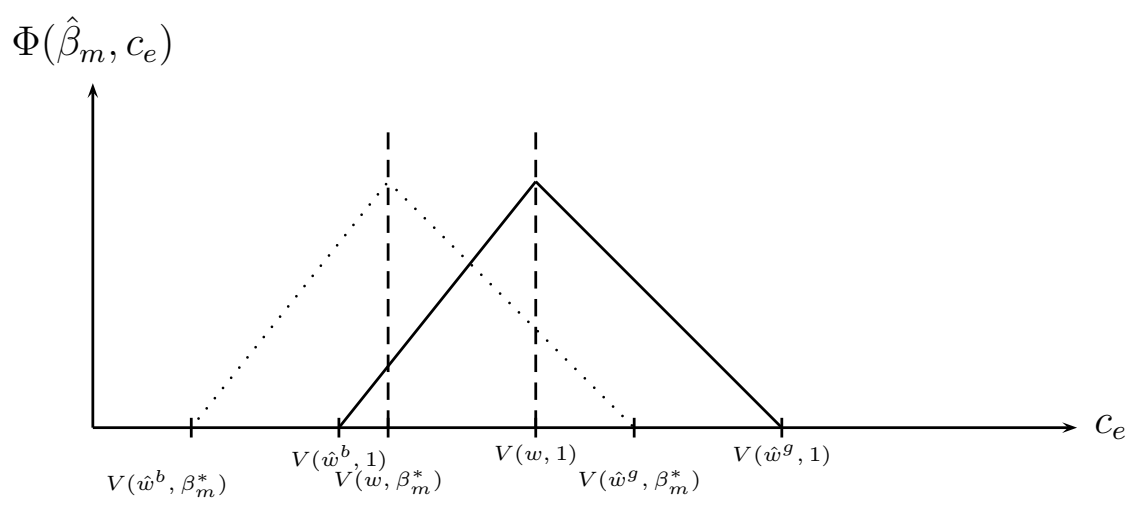

Figure 3: Sketch of the shift in the value of the decision maker's signal due to the expert's adjustment of his behavior to preselection.

(iv) If $c_{e} \geq V\left(\hat{w}^{g}, 1\right)$, the equilibrium is without preselection. The decision maker rejects the project without acquiring any information.

In these parameter constellations, in which the expert's advice does not depend on whether or not the decision maker has had a look at the project before asking for advice, the option to preselect strictly increases the decision maker's utility. In fact, there is a parameter region in which there is no information acquisition at all if the decision maker's signal is too expensive (if $c_{e} \geq V\left(w, \beta_{m}\right)$ and $\left.c_{d} \geq \Phi\left(\beta_{m}, c_{e}\right)\right)$. As the cost of the decision maker's signal drops sufficiently, both information sources are accessed with a positive probability, and the decision maker is willing to ask for the expert's advice at a higher cost as her own signal becomes cheaper.

Next, consider the parameter region where the expert's behavior depends on whether or not the project has been preselected by the decision maker. If $q \leq \frac{\left(\hat{w}^{g}\right)^{2}-\left(1-\hat{w}^{g}\right)^{2}\left(\frac{p_{m}}{1-p_{m}}\right)^{2}}{\left(\hat{w}^{g}\right)^{2}+\left(1-\hat{w}^{g}\right)^{2}\left(\frac{p_{m}}{1-p_{m}}\right)}, \beta_{m}=\beta_{m}^{*}<1$ (where $\beta_{m}^{*}$ solves $(2)$ ) if the expert anticipates preselection, but $\beta_{m}=1$ if he anticipates no preselection. The value of the expert's advice is lower in a putative equilibrium with preselection than in a putative equilibrium without preselection (see Lemma 3).

Proposition 2 implies that in a putative equilibrium without preselection, the value of the decision maker's signal is lower than in a putative equilibrium with preselection if the expert's advice is relatively cheap, and vice versa if the expert's advice is relatively expensive. Hence, the triangle depicted in Figure 2 shifts to the left as the decision maker anticipates the expert's reaction to her preselection behavior, as sketched in Figure $3{ }^{12}$

So far, there was no need to discuss the role of the expert's beliefs off the equilibrium path, because his best response does not depend on the decision maker's behavior (neither on nor off the equilibrium path) in the previously considered parameter range. Now, the expert's off-the-

\footnotetext{
${ }^{12}$ In Figure 3, the shape of the triangles are sketched as identical. Depending on the parameters, the dashed triangle can be steeper or flatter than the solid one.
} 
equilibrium-path-beliefs may be relevant for the equilibrium play. We discuss the role of the expert's beliefs off the equilibrium path (i.e., in the event of being asked for advice when this should never happen in equilibrium) in the proof of Proposition 4 in the appendix.

We distinguish four parameter ranges.

(i) For any $c_{e}$, if $c_{d}>\max \left\{\Phi\left(1, c_{e}\right), \Phi\left(\beta_{m}^{*}, c_{e}\right)\right\}$, the decision maker's signal is too expensive, independently of the expert's behavior. There is no preselection in equilibrium. If $c_{e}<V(w, 1)$, the expert is asked for advice. Else, the project is rejected without gathering information. In this case, being asked for advice occurs only off the equilibrium path.

(ii) For $\left(c_{e}, c_{d}\right)$ such that $c_{d}$ is smaller than $\Phi\left(\beta_{m}^{*}, c_{e}\right)$, but larger than $\Phi\left(1, c_{e}\right)$ (i.e., for a relatively low value of $c_{e}$, see Figure 3 ), there is an equilibrium with preselection and an equilibrium without preselection in which the decision maker always asks for the expert's advice. ${ }^{13}$ If the expert gives sincere advice (only if he expects the decision maker not to preselect), his advice is valuable enough such that the decision maker does not want to acquire an own signal. If the expert expects the decision maker to preselect, and hence distorts his advice, the decision maker indeed wants to acquire an own signal, because the value of the expert's advice deteriorates so much that she needs her own signal to assess whether it is worth to ask for advice.

(iii) For $\left(c_{e}, c_{d}\right)$ such that $c_{d}<\min \left\{\Phi\left(1, c_{e}\right), \Phi\left(\beta_{m}^{*}, c_{e}\right)\right\}$, preselection is attractive for the decision maker even though the expert adjusts his behavior. Due to the distortion in the expert's advice, the value of the expert's advice (when being asked) drops (see Lemma 3). As a consequence, the value of the decision maker's signal may either drop or increase. There are $c_{e}$ in the relevant range such that with sincere advice, the next-preferred alternative to preselection is to always ask for advice, whereas with distorted advice, the next-preferred alternative to preselection is to reject the project straight away. For low values of $c_{e}$ in this parameter range, the value of the decision maker's signal is enhanced by the expert's strategic adjustment of his advice, as the purpose of the signal acquisition is to sometimes avoid asking for advice. For higher values of $c_{e}$, where the advice is so costly that the decision maker only asks for it when she has observes a good signal herself, the value of her signal deteriorates with the expert's strategic distortion of advice.

(iv) For $\left(c_{e}, c_{d}\right)$ such that $c_{d}>\Phi\left(\beta_{m}^{*}, c_{e}\right)$ and $c_{d}<\Phi\left(1, c_{e}\right)$ (i.e., for relatively high values of $c_{e}$ ), the decision maker would like to preselect if the expert gives sincere advice, but not if the

\footnotetext{
${ }^{13}$ If $\Phi\left(\beta_{m}^{*}, c_{e}\right)$ and $\Phi\left(1, c_{e}\right)$ intersect, they do so at the upwards sloping part of $\Phi\left(1, c_{e}\right)$, i.e., for $c_{e}<V(w, 1)$, where the decision maker prefers to ask for the expert's advice if she does not observe her own signal and believes that $\beta_{m}=1$.
} 
advice is distorted. In fact, if the advice is distorted, the decision maker prefers not to acquire any information at all and to reject the project straight away. ${ }^{14}$ Hence, there is an equilibrium in which the decision maker rejects the project without acquiring information. If the expert is asked for advice, he believes that the decision maker has previously observed a good signal and distorts the advice. Uniqueness of this equilibrium behavior will be shown in the appendix.

We summarize the characterization of the equilibrium for the studied parameter range in the following proposition.

Proposition 4 Consider $q \leq \frac{\left(\hat{w}^{g}\right)^{2}-\left(1-\hat{w}^{g}\right)^{2}\left(\frac{p_{m}}{1-p_{m}}\right)^{2}}{\left(\hat{w}^{g}\right)^{2}+\left(1-\hat{w}^{g}\right)^{2}\left(\frac{p_{m}}{1-p_{m}}\right)} . \beta_{m}^{*}$ is the value which solves (2).

(i) Consider $\left(c_{e}, c_{d}\right)$ such that $c_{d}>\max \left\{\Phi\left(1, c_{e}\right), \Phi\left(\beta_{m}^{*}, c_{e}\right)\right\}$. In equilibrium, there is no preselection. If $c_{e}<V(w, 1)$, the expert is asked for advice. Else, the project is rejected without gathering information. $\beta_{m}=1$.

(ii) Consider $\left(c_{e}, c_{d}\right)$ such that $c_{d}<\Phi\left(\beta_{m}^{*}, c_{e}\right)$ and $\left.c_{d}>\Phi\left(1, c_{e}\right)\right)$. There are two equilibria, an equilibrium with preselection and an equilibrium without preselection in which the decision maker always asks for the expert's advice. In the former, $\beta_{m}=\beta_{m}^{*}$, in the latter, $\beta_{m}=1$.

(iii) Consider $\left(c_{e}, c_{d}\right)$ such that $c_{d}<\min \left\{\Phi\left(1, c_{e}\right), \Phi\left(\beta_{m}^{*}, c_{e}\right)\right\}$. There is a unique equilibrium with preselection. $\beta_{m}=\beta_{m}^{*}$.

(iv) Consider $\left(c_{e}, c_{d}\right)$ such that $c_{d}>\Phi\left(\beta_{m}^{*}, c_{e}\right)$ and $c_{d}<\Phi\left(1, c_{e}\right)$. There is a unique equilibrium in which the decision maker rejects the project without acquiring information.

\subsection{Decision quality}

We can now address the question in how far the decision quality is affected by the availability of a preselection stage when taking into consideration strategic adjustments on part of the expert. For the parameter constellation under consideration, if the ex ante reputation of the expert is high enough, his behavior does not depend on the availability of a preselection stage. In such a case, the additional information processing option is unambiguously beneficial for the decision maker.

If the expert adjusts his advice to the preselection behavior of the decision maker, the decision maker receives worse advice if she preselects than if she does not preselect. The advice to execute the project is more often based on mediocre information. On the other hand, if the decision maker asks for advice, the project is on average better. The effect on the decision

\footnotetext{
${ }^{14}$ If $\Phi\left(\beta_{m}^{*}, c_{e}\right)$ and $\Phi\left(1, c_{e}\right)$ intersect, they do so at the downwards sloping part of $\Phi\left(\beta_{m}^{*}, c_{e}\right)$, i.e., for $c_{e}>$ $V\left(w, \beta_{m}^{*}\right)$, where the decision maker prefers to reject the project if she does not observe her own signal and believes that $\beta_{m}=\beta_{m}^{*}$.
} 


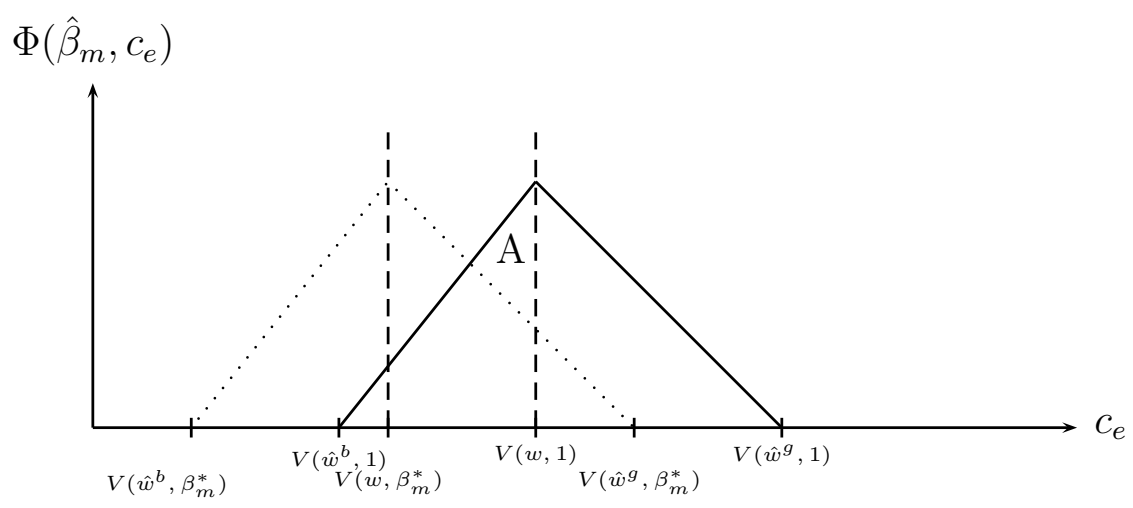

Figure 4: In the parameter region $\mathrm{A}$, the decision maker rejects the project without acquiring information, anticipating low-quality advice. If the decision maker does not have the option to preselect, the expert gives sincere advice, which is valuable to the decision maker.

quality depends on the relative quality of information processing at both stages.

We identify parameter constellations such that the option to preselect yields the unconditional rejection of the project in any equilibrium, whereas the expert would always be consulted if the decision maker does not have access to a (cheap enough) own signal. In these cases, the decision maker is clearly worse off. These parameter constellations are shown in Figure 4.

If it is worthwhile to acquire the expert's advice if the mediocre expert is sincere, but not if his advice is distorted, then the access to a cheap signal is detrimental for the decision maker. Note that the adjustment of the expert's reporting behavior reinforces the decision maker's incentive to acquire an own signal (see Proposition 2 (i)). However, in equilibrium, there is no information acquisition at all. Paradoxically, a decrease in the cost of information acquisition (a drop in $c_{d}$ ) eliminates the decision maker's demand for information.

\section{Conclusion}

Due to scarce refereeing resources, many academic journals apply (or consider to apply) deskrejection policies. That is, an editor seeks a referee's advice for a publication decision only after having scanned the paper herself and assessed its quality as sufficiently high. Otherwise, the paper is rejected right away. With a desk-rejection policy, the journal risks sorting out good papers that at first glance look mediocre. Our analysis suggests that with this practice, the journal may also accept mediocre papers more often. In fact, both mistakes, accepting mediocre papers and rejecting good ones may become more likely when a journal implements a desk-rejection policy. Consider, for example, a parameter constellation for which in equilibrium a mediocre referee never recommends the publication of a paper if no desk-rejection policy is in place due to low quality expectations. Suppose that a desk-rejection policy sufficiently boost 
his quality expectations such that he sometimes recommends the publication of the paper. As a consequence, when a paper is accepted for publication, it is more likely to be mediocre than without a desk-rejection policy. If at the same time desk-rejection decisions rely on relatively weak signals, it gets also more likely that a rejected paper is in fact good.

More generally, our analysis suggests to be cautious when introducing an additional information processing layer in an organization with career-concerned agents. Such an additional layer can be particularly harmful when the cost of the expert's advice is comparatively high. If the adjustment of the expert's behavior to preselection causes the value of advice to drop below its cost, information processing on the second layer becomes infeasible, and may even render the information processing on the first layer worthless.

Due to the advancements in information availability and information processing technologies, it becomes cheaper to obtain a first assessment of a decision problem. As a consequence, decision makers may be tempted to pre-process a problem before asking an expert for advice. As this paper illustrates, the adjustment of the expert's behavior on a later stage of information processing may outweigh the cost advantage on the first stage of information processing.

\section{References}

[1] AVERY, C. and CHEVALIER, J. (1999): "Herding over the career", Economic Letters, 63, 327-333.

[2] BENABOU, R. and LAROQUE, G. (1992): "Using privileged information to manipulate markets: insiders, gurus, and credibility", Quarterly Journal of Economics, 107, No. 3, 921-958.

[3] CHEN, Y. (2015): "Career concerns and excessive risk taking", Journal of Economics and Management Strategy, 24, No. 1, 110-130.

[4] EFFINGER, M. R. and POLBORN, M. K. (2001): "Herding and anti-herding: A model of reputational di!erentiation", European Economic Review, 45, 385-403.

[5] FELGENHAUER, M. and SCHULTE, E. (2014): "Strategic private experimentation", American Economic Journal: Microeconomics, 6, No. 4, 74-105.

[6] FOX, J. and VAN WEELDEN, R. (2010): "Partisanship and the effectiveness of oversight", Journal of Public Economics, 94, No. 9-10, 684-697. 
[7] FU, Q. and LI, M. (2014): "Reputation-concerned policy makers and institutional status quo bias", Journal of Public Economics, 110, 15-25.

[8] HOLMSTRÖM, B. (1999): "Managerial incentive problems: A dynamic perspective", Review of Economic Studies, 66, 169-182.

[9] KARTIK, N., LEE, F. X. and SUEN, W. (2016): "Investment in concealable information by biased experts", RAND Journal of Economics, forthcoming.

[10] KREPS, D. M. and WILSON, R. (1982): "Sequential Equilibria", Econometrica, 50, 863894.

[11] LAGERLÖF, J. and FRISELL, L. (2007): "A model of reputation in cheap talk", Scandinavian Journal of Economics, 109, No. 1, 49-70.

[12] LIU, Y. F. and SANYAL, A. (2012): "When second opinions hurt: A model of expert advice under career concerns", Journal of Economic Behavior $\& 6$ Organization, 84, 1-16.

[13] LEAVER, C. (2009): "Bureaucratic minimal squawk behavior: theory and evidence from regulatory agencies", American Economic Review, 99, No. 3, 572-607.

[14] LEVY, G. (2004): "Anti-herding and strategic consultation", European Economic Review, $48,503-525$.

[15] LEVY, G. (2005): "Careerist judges and the appeals process", RAND Journal of Economics, 36, No. 2, 275-297.

[16] MilbOURN, T. T., SHOCKLEY, R. L. and THAKOR, A. V. (2001): "Managerial career concerns and investments in information", RAND Journal of Economics, 32, No. 2, 334351.

[17] MORRIS, S. (2001): "Political correctness", Journal of Political Economy, 109, No. 2, 231-265.

[18] OTTAVIANI, M. and SØRENSEN, P (2006): "Professional advice", Journal of Economic Theory, 126, 120-142.

[19] OTTAVIANI, M. and SØRENSEN, P (2001): "Information aggregation in debate: who should speak first?", Journal of Public Economics, 81, 393-421. 
[20] PRAT, A. (2005): "The wrong kind of transparency", American Economic Review, 95, No. 3, 862-877.

[21] SCHARFSTEIN, D. and STEIN, J. (1990): "Herd behavior and investment", American Economic Review, 80, No. 3, 465-479.

[22] SOBEL, J. (1985): "A theory of credibility", Review of Economic Studies, 52, 557-573.

[23] SUURMOND, G., VISSER, B. and SWANK, O. H. (2004): "On the bad reputation of reputational concerns", Journal of Public Economics, 88, 2817-2838.

[24] SWANK, O. H. and VISSER, B. (2008): "The consequences of endogenizing information for the performance of a sequential decision structure", Journal of Economic Behavior and Organization, 65, 667-681.

\section{Appendix}

Comment on the restriction to pure strategies on part of the decision maker. It is harmless to rule out randomization on part of the decision maker when consulting the expert in an equilibrium with preselection: It is not optimal for the decision maker to acquire a signal and to randomize after its realization. To see this, suppose to the contrary that she does so in equilibrium. Bear in mind that in order to randomize over her actions, the decision maker needs to be indifferent whether or not to consult the expert after the observation of her signal at least for one of its possible realizations. As her own signal is informative, she can only be indifferent for one realization of the signal. For the other realization, she strictly prefers one action over the other. By choosing this action immediately, without the acquisition of a costly signal, she obtains a higher payoff. ${ }^{15}$

Regarding the decision whether to preselect, randomization may be optimal if the value of information acquired at this stage is equal to its cost. If such a randomization is optimal, it is also optimal for the decision maker (i) to consult the expert in the case that she did not acquire a signal, and (ii) to make the consultation choice dependent on the signal realization if she has acquired a signal. If such a behavior occurs in equilibrium, the expert cannot perfectly deduce the decision maker's belief about the state of the world, which makes the analysis more involved (such an analysis was carried out in a previous version of the paper, which is available

\footnotetext{
${ }^{15}$ In an equilibrium without preselection, randomization can occur at the stage of consulting the expert, but it will not induce any behavioral response by the expert, who only acts conditional on being consulted.
} 
upon request). Recall that the value of information depends on the expert's behavior. With a slight perturbation of the expert's behavior, the indifference at the preselection stage breaks down.

Proof of Lemma 1. (i) For all $\sigma_{d}$ and $q>1 / 2, \hat{w}_{Y}^{\sigma_{d}}>\hat{w}_{N}^{\sigma_{d}}$ independently of the mediocre expert's behavior. Bayesian plausibility requires $\hat{w}_{N}^{\sigma_{d}}<\hat{w}^{\sigma_{d}}$. By assumption, $\hat{w}^{g}<\Delta_{b} /\left(\Delta_{b}+\Delta_{g}\right)$. As $\hat{w}^{g}>\hat{w}^{\emptyset}=w>\hat{w}^{b}$, we have $\hat{w}_{N}^{\sigma_{d}}<\Delta_{b} /\left(\Delta_{b}+\Delta_{g}\right)$ for all $\sigma_{d}$. Suppose, contrary to the claim in the lemma, that the expert is consulted but $\hat{w}_{Y}^{\sigma_{d}} \leq \Delta_{b} /\left(\Delta_{b}+\Delta_{g}\right)$. If the strict inequality holds, the decision maker strictly prefers to reject the project. For $\hat{w}_{Y}^{\sigma_{d}}=\Delta_{b} /\left(\Delta_{b}+\Delta_{g}\right)$, the decision maker obtains the same expected utility when executing or rejecting the project, hence assume that she rejects it. As $\hat{w}_{N}^{\sigma_{d}}<\Delta_{b} /\left(\Delta_{b}+\Delta_{g}\right)$, she rejects the project when the expert recommends to do so. Thus, before asking the expert for advice, the decision maker anticipates that she rejects the project independently of his advice. At that stage, her expected utility is $U_{N}^{d}\left(\hat{w}^{\sigma_{d}}\right)-c_{e}$. She is better off rejecting the project without consulting the expert, saving the $\operatorname{cost} c_{e}$. (ii) follows.

Q.E.D.

Proof of Lemma 2. By definition, in an equilibrium with preselection, the decision maker acquires the signal $\sigma_{d}$. In such an equilibrium, the expert needs to be consulted sometimes. It is not optimal for the decision maker to acquire the signal $\sigma_{d}$, but never to consult the expert. Her own signal cannot convince her to execute the project, hence the project is always rejected if the expert is not consulted. The decision maker is better off saving the cost of information acquisition $c_{d}$.

Due to Lemma 1, the decision maker follows the expert's advice if she asks for it. Without consulting the expert, the decision maker rejects the project. Hence, the value of consulting the expert given the signal $\sigma_{d}$ is:

$$
\begin{aligned}
& \operatorname{prob}\left\{\omega=g \mid \sigma_{d}\right\}\left(\operatorname{prob}\left\{x_{e}=Y \mid \omega=g\right\} u(Y, g)+\operatorname{prob}\left\{x_{e}=N \mid \omega=g\right\} u(N, g)\right) \\
+ & \operatorname{prob}\left\{\omega=b \mid \sigma_{d}\right\}\left(\operatorname{prob}\left\{x_{e}=Y \mid \omega=b\right\} u(Y, b)+\operatorname{prob}\left\{x_{e}=N \mid \omega=b\right\} u(N, b)\right) \\
- & \operatorname{prob}\left\{\omega=g \mid \sigma_{d}\right\} u(N, g)-\operatorname{prob}\left\{\omega=b \mid \sigma_{d}\right\} u(N, b) \\
=\quad & \hat{w}^{\sigma_{d}} \operatorname{prob}\left\{x_{e}=Y \mid \omega=g\right\}(u(Y, g)-u(N, g)) \\
+ & \left(1-\hat{w}^{\sigma_{d}}\right) \operatorname{prob}\left\{x_{e}=Y \mid \omega=b\right\}(u(Y, b)-u(N, b)) \\
=\quad & \hat{w}^{\sigma_{d}} \operatorname{prob}\left\{x_{e}=Y \mid \omega=g\right\} \Delta_{g}-\left(1-\hat{w}^{\sigma_{d}}\right) \operatorname{prob}\left\{x_{e}=Y \mid \omega=b\right\} \Delta_{b}
\end{aligned}
$$

We have $\Delta_{g}>0, \Delta_{b}>0$. Hence, (6) is increasing in $\hat{w}^{\sigma_{d}}$. As $\hat{w}^{g}>\hat{w}^{b}$, the value of consulting the expert is higher when the decision maker has observed $\sigma_{d}=g$ than when she has observed $\sigma_{d}=b$. Thus, if the decision maker consults the expert for some signal, she consults him if 
$\sigma_{d}=g$. This proves the first part ("if") of the statement.

To prove the second part ("only if"), we need to rule out the case that the decision maker consults the expert for both realizations of $\sigma_{d}$. Suppose she does so. Then, for some recommendation $Y$ or $N$, the project choice for $\sigma_{d}=g$ must differ from that for $\sigma_{d}=b$. Otherwise, no action depends on $\sigma_{d}$ such that the decision maker is better off saving the cost $c_{d}$. However, that implies that the decision maker sometimes does not follow the expert's advice, a contradiction to Lemma 1. Hence, we obtain a contradiction to the assumption that the expert is always consulted, which completes the proof.

Q.E.D.

Proof of Proposition 1. (i) Please refer to the online appendix for a full analysis of the expert's putative equilibrium behavior. Given the belief that the expert holds about the decision maker's preselection behavior, there is a unique combination of mutually consistent behavior $\gamma_{m}, \beta_{m}$ of the expert and beliefs $\hat{\gamma}_{m}, \hat{\beta}_{m}$ on part of the decision maker. In the light of Lemma 2, there are only two putative equilibrium beliefs about the preselection behavior. In the case of preselection, conditional on being asked for advice, the expert believes that the decision maker has observed a good signal. In the case of no preselection, the expert believes that the decision maker has not acquired any information. Uniqueness of the expert's possible equilibrium behavior in the respective cases (if such a preselection behavior occurs in equilibrium) follow from Lemma A7 in the online appendix.

(ii) Denote with $\hat{w}_{m}$ the mediocre expert's assessment of the probability that $\omega=g$ (conditional on his own signal $\sigma_{m}$ and the belief about the decision maker's signal) when giving a recommendation.

We first derive the thresholds for $q$ such that an equilibrium with $\gamma_{m}=\beta_{m}=1$ exists if and only if $q$ is between the thresholds. If $\hat{\gamma}_{m}=\hat{\beta}_{m}=1$, it is optimal for the expert to recommend the execution of the project if and only if: ${ }^{16}$

$$
\frac{\hat{w}_{m} q}{q+(1-q) p_{m}} \geq \frac{q\left(1-\hat{w}^{\sigma_{d}}\right)}{q\left(1-\hat{w}^{\sigma_{d}}\right)+(1-q)\left(\hat{w}^{\sigma_{d}}\left(1-p_{m}\right)+\left(1-\hat{w}^{\sigma_{d}}\right) p_{m}\right)},
$$

which is equivalent to:

$$
q \leq \frac{\hat{w}_{m} \hat{w}^{\sigma_{d}}\left(1-p_{m}\right)-\left(1-\hat{w}_{m}\right)\left(1-\hat{w}^{\sigma_{d}}\right) p_{m}}{\left(\left(1-\hat{w}_{m}\right)\left(1-\hat{w}^{\sigma_{d}}\right)+\hat{w}_{m} \hat{w}^{\sigma_{d}}\right)\left(1-p_{m}\right)} .
$$

In equilibrium, the expert correctly anticipates the decision maker's signal. ${ }^{17}$ Conditional on $\sigma_{m}=g$, we have $\hat{w}_{m}=\frac{\hat{w}^{\sigma} d p_{m}}{\hat{w}^{\sigma} d p_{m}+\left(1-\hat{w}^{\sigma}\right)\left(1-p_{m}\right)}$. Conditional on $\sigma_{m}=b$, we have $\hat{w}_{m}=$ $\frac{\hat{w}^{\sigma} d\left(1-p_{m}\right)}{\hat{w}^{\sigma} d\left(1-p_{m}\right)+\left(1-\hat{w}^{\sigma} d\right) p_{m}}$.

\footnotetext{
${ }^{16}$ In order to avoid qualifications in our statement, we break indifference in favor of recommending the execution of the project.

${ }^{17}$ Remember that we exclude equilibria in which the decision maker randomly acquires a signal.
} 
With $\sigma_{m}=g,(7)$ holds if

$$
q \leq \frac{\left(\hat{w}^{\sigma_{d}}\right)^{2}-\left(1-\hat{w}^{\sigma_{d}}\right)^{2}}{\left(\hat{w}^{\sigma_{d}}\right)^{2}+\left(1-\hat{w}^{\sigma_{d}}\right)^{2}\left(\frac{1-p_{m}}{p_{m}}\right)}:=\bar{q} .
$$

The mediocre expert's best response is to truthfully reveal $\sigma_{m}=g$, if and only if $q \leq \bar{q}$.

Finally, $\beta_{m}=1$ is a best response if and only if (7) does not hold for $\sigma_{m}=b$. This is the case if

$$
q>\frac{\left(\hat{w}^{\sigma_{d}}\right)^{2}-\left(1-\hat{w}^{\sigma_{d}}\right)^{2}\left(\frac{p_{m}}{1-p_{m}}\right)^{2}}{\left(\hat{w}^{\sigma_{d}}\right)^{2}+\left(1-\hat{w}^{\sigma_{d}}\right)^{2}\left(\frac{p_{m}}{1-p_{m}}\right)}:=\underline{q} .
$$

If $q \in(\underline{q}, \bar{q}]$, then the expert's equilibrium response to the decision maker's preselection behavior (captured by $\hat{w}^{\sigma_{d}}$ ) is $\gamma_{m}=\beta_{m}=1$. If $q<\underline{q}$, then the incentive to recommend the execution of the project is so strong that the expert does so with a positive probability even if he has observed a bad signal. According to Lemma A2 in combination with Lemma A3 in the online appendix, in equilibrium $\gamma_{m}=1$ and $0<\beta_{m}<1$. If $q>\bar{q}$, then the incentive to recommend the rejection of the project is so strong that the expert does so with a positive probability even if he has observed a good signal. In equilibrium, $\beta_{m}=1, \gamma_{m}<1$.

The last step is to derive the threshold for $q$ beyond which the safe haven is so attractive that the mediocre expert never recommends to execute the project. In this case $\hat{q}_{Y}^{g}=1 .^{18}$ The incentive to deviate from the suggested behavior is strongest if $\sigma_{m}=g \cdot \gamma_{m}=0, \beta_{m}=1$ is equilibrium play if and only if

$$
\frac{\hat{w}^{\sigma_{d}} p_{m}}{\hat{w}^{\sigma_{d}} p_{m}+\left(1-\hat{w}^{\sigma_{d}}\right)\left(1-p_{m}\right)}<\frac{q\left(1-\hat{w}^{\sigma_{d}}\right)}{q\left(1-\hat{w}^{\sigma_{d}}\right)+(1-q)}
$$

i.e., if and only if

$$
q>\frac{\hat{w}^{\sigma_{d}} p_{m}}{\hat{w}^{\sigma_{d}} p_{m}+\left(1-\hat{w}^{\sigma_{d}}\right)^{2}\left(1-p_{m}\right)}:=\tilde{q}
$$

Q.E.D.

\section{Restricting the parameter range}

The lower bound of the interval $\left(\frac{w^{2}-(1-w)^{2}\left(\frac{p_{m}}{1-p_{m}}\right)^{2}}{w^{2}+(1-w)^{2}\left(\frac{p_{m}}{1-p_{m}}\right)}, \frac{w^{2}-(1-w)^{2}}{w^{2}+(1-w)^{2}\left(\frac{1-p_{m}}{p_{m}}\right)}\right]$ decreases in $p_{m}$ whereas the upper bound increases in $p_{m}$. Remember that we assume $q>1 / 2$. The upper bound of the interval is greater than $1 / 2$ if $w>1 / 2$ and $p_{m}>(1-w)^{2} /(2 w-1)$. It is greater than $1 / 2$

\footnotetext{
${ }^{18}$ The joint observation of $x_{e}=Y$ and $\omega=b$ occurs only off the equilibrium path. We assume that the decision maker attributes such an observation to a deviation by the mediocre type. Consequently, $\hat{q}_{Y}^{b}=0$. Other offthe-equilibrium-path-beliefs may support the same equilibrium if $q$ is sufficiently large. They cannot give rise to different equilibrium actions on the equilibrium path. At the threshold, the admissable out-of-equilibrium-beliefs are unique.
} 
for all $p_{m} \geq 1 / 2$ if $w>3 / 2-\sqrt{3 / 4}$. Thus, for $w>3 / 2-\sqrt{3 / 4}$ and for all $p_{m}>1 / 2$, we can identify a non-empty interval $Q$ such that if $q \in Q$, then the expert gives sincere advice in an equilibrium without preselection.

Summarizing the parameter restrictions:

- $q>1 / 2$

- $w>3 / 2-\sqrt{3 / 4}$

- $q \in\left(\frac{w^{2}-(1-w)^{2}\left(\frac{p_{m}}{1-p_{m}}\right)^{2}}{w^{2}+(1-w)^{2}\left(\frac{p_{m}}{1-p_{m}}\right)}, \frac{w^{2}-(1-w)^{2}}{w^{2}+(1-w)^{2}\left(\frac{1-p_{m}}{p_{m}}\right)}\right]$

- $\Delta_{b} \in\left(\frac{\hat{w}^{g}}{1-\hat{w}^{g}} \Delta_{g}, \frac{w\left(q+(1-q) p_{m}\right)}{(1-w)(1-q)\left(1-p_{m}\right)} \Delta_{g}\right]$

Derivation of $V\left(\hat{w}^{\sigma_{d}}, \hat{\beta}_{m}\right)$

$$
\begin{aligned}
V\left(\hat{w}^{\sigma_{d}}, \hat{\beta}_{m}\right)= & \hat{w}^{\sigma_{d}}\left(q+(1-q)\left(p_{m}+\left(1-p_{m}\right)\left(1-\beta_{m}\right)\right)\right) u(Y, g) \\
& +\hat{w}^{\sigma_{d}}\left(1-\left(q+(1-q)\left(p_{m}+\left(1-p_{m}\right)\left(1-\beta_{m}\right)\right)\right)\right) u(N, g) \\
& -\hat{w}^{\sigma_{d}} u(N, g) \\
& +\left(1-\hat{w}^{\sigma_{d}}\right)\left(q+(1-q) p_{m} \beta_{m}\right) u(N, b) \\
& +\left(1-\hat{w}^{\sigma_{d}}\right)\left(1-\left(q+(1-q) p_{m} \beta_{m}\right)\right) u(Y, b) \\
& -\left(1-\hat{w}^{\sigma_{d}}\right) u(N, b) \\
= & \hat{w}^{\sigma_{d}}\left(q+(1-q)\left(p_{m}+\left(1-p_{m}\right)\left(1-\beta_{m}\right)\right)\right) \Delta_{g} \\
& +\left(1-\hat{w}^{\sigma_{d}}\right)\left(q+(1-q) p_{m} \beta_{m}\right) \Delta_{b} \\
& -\left(1-\hat{w}^{\sigma_{d}}\right) \Delta_{b} \\
= & \hat{w}^{\sigma_{d}}\left(q+(1-q) p_{m}\right) \Delta_{g}-\left(1-\hat{w}^{\sigma_{d}}\right)(1-q)\left(1-p_{m}\right) \Delta_{b} \\
& +\left(1-\hat{\beta}_{m}\right)(1-q)\left(\hat{w}^{\sigma_{d}}\left(1-p_{m}\right) \Delta_{g}-\left(1-\hat{w}^{\sigma_{d}}\right) p_{m} \Delta_{b}\right) .
\end{aligned}
$$

Proof of Proposition 2. (i) Taking the partial derivative of (4) with respect to $\hat{\beta}_{m}$ yields:

$$
w\left(1-p_{d}\right)(1-q)\left(1-p_{m}\right) \Delta_{g}-(1-w) p_{d}(1-q) p_{m} \Delta_{b}
$$

This expression is negative if $w\left(1-p_{d}\right)(1-q)\left(1-p_{m}\right) \Delta_{g}<(1-w) p_{d}(1-q) p_{m} \Delta_{b}$, i.e., if $\Delta_{b}>\frac{w\left(1-p_{d}\right)\left(1-p_{m}\right)}{(1-w) p_{d} p_{m}} \Delta_{g}$, where the right-hand-side equals $\frac{\hat{w}^{b}\left(1-p_{m}\right)}{\left(1-\hat{w}^{b}\right) p_{m}} \Delta_{g}$, which is smaller than $\frac{\hat{w}^{g}}{\left(1-\hat{w}^{g}\right)} \Delta_{g}$. Thus, the inequality is implied by the assumption that $\Delta_{b}>\frac{\hat{w}^{g}}{\left(1-\hat{w}^{g}\right)} \Delta_{g}$.

(ii) Taking the partial derivative of $(5)$ with respect to $\hat{\beta}_{m}$ yields:

$$
-w p_{d}(1-q)\left(1-p_{m}\right) \Delta_{g}+(1-w)\left(1-p_{d}\right)(1-q) p_{m} \Delta_{b}
$$


This expression is positive if $\Delta_{b}>\frac{w p_{d}\left(1-p_{m}\right)}{(1-w)\left(1-p_{d}\right) p_{m}} \Delta_{g}$, which is once more implied by the assumption that $\Delta_{b}>\frac{\hat{w}^{g}}{\left(1-\hat{w}^{g}\right)} \Delta_{g}$.

Proof of Proposition 3 and 4. Proposition 3 follows directly from the arguments in the text. For Proposition 4, we complement the arguments provided in the main text with a discussion of out-off-equilibrium-beliefs on part of the expert, and we show uniqueness of the equilibrium as claimed in Proposition 4 (iv).

(i) It does not matter for equilibrium play whether the expert believes that the decision maker asks for his advice (off the equilibrium path) after having observed a good signal herself or without having acquired an own signal, because neither belief induces a behavior that makes it worthwhile for the decision maker to ask for his advice. Therefore, any such beliefs support the unique equilibrium behavior.

(ii) and (iii) In these parameter ranges, there is no off-the-equilibrium-path-event.

(iv) In any putative equilibrium in which the expert believes that the decision maker has not preselected (whether on or off the equilibrium path), and, hence, would give sincere advice if asked for it, the decision maker has a strict incentive to preselect, as the value of doing so is positive if the advice is sincere. Consequently, the expert's belief may potentially only be compatible with equilibrium play if it is held off the equilibrium path, implying that the expert is not asked for advice along the equilibrium path.

For completeness of the analysis, suppose that upon being asked for advice, the expert believes that the decision maker acquires a signal with a probability strictly smaller than one and asks for the expert's advice if uninformed or after having observed a good signal. This belief induces a smaller distortion in the expert's advice. By varying the probability of the signal acquisition by the decision maker, and having the expert respond accordingly, the triangle capturing the value of the decision maker's signal and the value of advice as depicted in Figure 2 shifts between the two triangles depicted in Figure 3. Note, however, that advice-seeking is worthwhile for the decision maker in the case of being uninformed only in parameter constellations where $c_{e}$ is smaller than the value for which $\Phi\left(\beta_{m}, c_{e}\right)$ reaches its peak. In that part, however, the value of the decision maker's signal increases as the experts starts distorting his advice (see Proposition 2). Hence, it is impossible for the expert in the parameter constellation under consideration to make the decision maker indifferent with respect to the signal acquisition. Consequently, an equilibrium with such beliefs in which the expert is asked for advice does not exist.

Q.E.D. 
This paper is published by Springer in the International Journal of Game Theory. DOI: $10.1007 / \mathrm{s} 00182-016-0551-9$

The final publication is available at http://link.springer.com/article/10.1007/s00182016-0551-9. 